\title{
Pharmacokinetic Parameters Result Standard Unit
}

National Cancer Institute

\section{Source}

National Cancer Institute. Pharmacokinetic Parameters Result Standard Unit. NCI

Thesaurus. Code C113378.

The standard unit of measure for pharmacokinetic parameters results. 